\title{
Millimeter Wave RF Front End Design using Neuro-Genetic Algorithms
}

\author{
Rana J. Pratap, J.H. Lee, S. Pinel, G.S. May ${ }^{*}$, J. Laskar and E.M. Tentzeris \\ Georgia Electronic Design Center \\ Georgia Institute of Technology, Atlanta, GA -30332-0250 \\ "Office: (404) 894-5053, FAX : (404) 894-5028, Email : gary.may@ece.gatech.edu
}

\begin{abstract}
This paper presents neural network and genetic algorithm based modeling and design of millimeter wave RF front end circuits. The neuro-genetic design methodology is composed of two stages. Stage one consists of the development of an accurate neural network model for the microwave filters from the measured data. This model can be used to perform sensitivity analysis and derive response surfaces. In the second stage, the neural network model is used in conjunction with genetic algorithms to synthesize millimeter wave devices with desired electrical specifications. The synthesis methodology uses an accurate model that accounts for the manufacturing variations and parameter indeterminacy issues. Furthermore, the genetic synthesis algorithm uses a priority scheme to account for tradeoffs among various electrical characteristics to provide the best design. This method has been used to synthesize mm-wave low pass and band pass filters. The electrical response obtained from the layout parameters predicted by the method matches the desired electrical characteristics within 5\%. The generic nature of the technique suggests potential extension to other mm-wave front ends, such as antennas, diplexers and baluns.
\end{abstract}

\section{Introduction}

Microwave filters are an important component in wireless communication systems. However, millimeter wave microwave component design poses great challenges due to the requirements of high frequency operation, where there is enhanced parasitic coupling, transmission, and radiation loss. The effects of metal roughness and variations in the dielectric constant also have significant impact on mm-wave circuit performance. Existing tools are limited in their capability to model the complex, nonlinear behavior of such devices [1]. There is significant variation in their measured and simulated behavior of due to the impact of manufacturing variations and modeling inaccuracies at high frequency. Therefore, there is need for a design methodology that uses measured data and accounts for manufacturing variations.

There are two aspects of microwave design. The first step is to derive an accurate model, and next step is to use the model to interactively synthesize devices with desired electrical characteristics. Several previous attempts have been made to model and synthesize microwave filters using various CAD methods. Most approaches combine finite element techniques with optimization methods like gradient descent and genetic algorithms [2-3]. However, such approaches are often tedious, since each iteration of the optimization process requires the simulation of a device structure with new sets of parameters using the EM simulator. Typically, hundreds of iterations are required to obtain a good solution. EM simulation methods are also inadequate because they require approximation, either in the description of the structure to be analyzed (metal roughness and surrounding environment) or in terms of boundary conditions. Finite element models are accurate, but time-consuming. Regression models are faster, but lack accuracy. On the other hand, analytical physical models are complex to derive and optimize. They also utilize simplifying assumptions that can reduce accuracy. Neural networks have emerged as an effective alternative for circuit modeling because of their greater accuracy and low computational cost. However, earlier studies have limited the use of neural networks to modeling and analysis and have not addressed the use of these models for design synthesis.

Compact, integrated, and low-cost filter design for optimal electrical performance continues to be a challenge for microwave designers. Filter design involves several trade-offs between various design parameters. There are always two or more performance parameters for which improvement in one leads to deterioration of the other parameters. For example, narrow bandwidth leads to greater pass band insertion loss and vice-versa. There is a significant need to determine a precise set of layout parameters that meets desired electrical specifications (such as operating frequency, bandwidth, insertion loss, etc.). The algorithm for synthesizing such a design should be highly accurate, but not overly timeconsuming.

In this paper, we present a neuro-genetic approach for design and optimization of millimeter wave low pass and bandpass filters. This method has been previously used for multilayer inductor and capacitor design [4]. The proposed methodology combines the accuracy of neural networks for nonlinear modeling with the efficiency of genetic algorithms in optimizing the parameter space. The methodology has two phases. In the first phase, a neural network model is developed from measured data. This model can be used to perform sensitivity analysis and obtain response surfaces. In the second phase, a genetic algorithm-based scheme is used in conjunction with the neural network models for filter design and synthesis. This unique coupling enables the identification of the correct combination of layout parameters to achieve desired electrical specifications. The proposed method also has the capability to prioritize multi-parameter optimization to suit specific filter design requirements. This capability gives additional flexibility to filter designers to accommodate tradeoffs and obtain the best available design.

\section{Neuro-Genetic Design Methodology}

\subsection{Neural Network Modeling}

Neural networks have emerged as an attractive technique for modeling complex nonlinear relationships [5]. Neural networks posses the capability to learn arbitrary mappings between noisy sets of input and output parameters. Neural 
network training is a self-organizing process designed to determine an appropriate set of connection weights that allow the activation of simple processing units to achieve a desired state of activation that mimics the relationship between a given set of samples.

The neural network used for modeling is a multilayer perceptron network consisting of three or more layers as shown in Figure 1. The x-y-z neural network structure refers to number of neurons in the input, hidden and output layers respectively. The network is typically trained using the error back-propagation (BP) algorithm with a hyperbolic tangent activation function [6]. The BP algorithm uses the gradient descent technique, which systematically changes the network weights by an amount proportional to the partial derivative of the accumulated error function, $E$, with respect to given weight. The change in weight is given by

$$
\Delta w_{i j k}=-\eta \frac{\partial E}{\partial w_{i j k}}
$$

where $i$ denotes a node in layer $k, j$ a node in preceding layer $(k-1)$, and $w_{i j k}$ the weight between these two nodes. The constant $\eta$ (which lies in the range $0-1$ ) is called the learning rate. The learning rate determines the speed of convergence by regulating the size of the weight change. A larger rate may result in the algorithm settling at a local minimum. A smaller rate can promote stability in the network, but results in longer training time. In order to improve training an additional momentum term can be added. The momentum term deters the algorithm from settling in local minima and increases the speed of convergence. The weight of the back propagation at the $(n+1)^{\text {th }}$ iteration is then given by:

$w_{i j k}(n+1)=w_{i j k}(n)+\eta \Delta w_{i j k}(n)+\alpha \Delta w_{i j k}(n-1)$

where $\alpha$ (also in the 0-1 range) is the momentum constant.

The accuracy of the model is evaluated in terms of the root-mean-square error (RMSE). The prediction RMSE is calculated by obtaining the square root error between the neural network's predicted value and actual value and is given by

$$
R M S E=\sqrt{\frac{1}{n-1} \sum_{i=1}^{n}\left(y_{i}-\hat{y}_{i}\right)^{2}}
$$

where $n$ is the number of trials and $y_{i}$ is the measured values of each response, and $\hat{y}_{i}$ is the neural model output. The training error is the RMSE of the data used for network training, and the prediction error is the RMSE of the data reserved for network testing.

Network structure and training issues, such as the number of layers, number of neurons, the learning rate and the momentum constant are determined during the model development process. These values are selected such that after training, the network model outputs best match the experimental data.

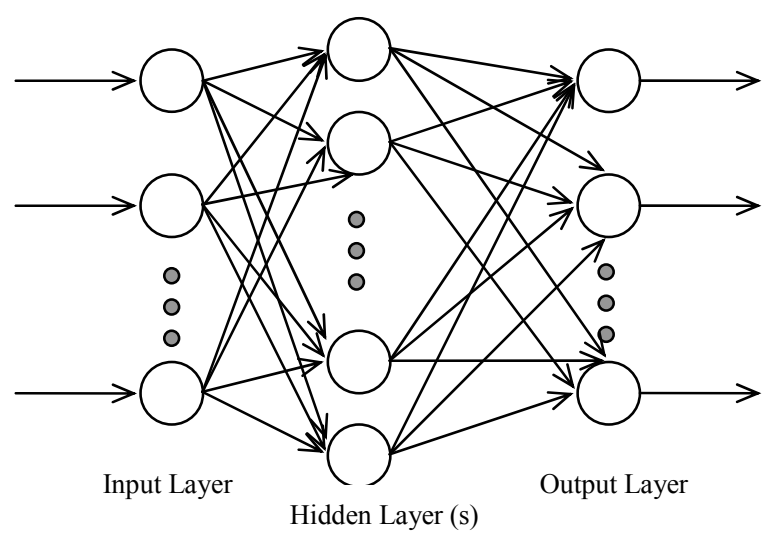

Figure 1. Multilayer perceptron neural network structure.

\subsection{Genetic Algorithms}

Genetic algorithms are a guided stochastic search technique based on mechanics of evolution and natural selection [7]. They operate by interactively cycling through creation of a "population" of strings, evaluation and selection of "most fit" strings, and genetic manipulation to create a new population. The strings are formed by encoding each variable into a series of binary bits. The new population is created by reproduction, crossover, and mutation. Reproduction is process by which strings with high fitness values (F) are selected based on a probabilistic approach known as elitist roulette wheel selection, which is described by:

$$
\begin{aligned}
& P_{\text {select } \_i_{i}}=\frac{F_{i}}{\sum F} \\
& F=\frac{1}{1+\sum_{n}\left|K_{n}\left(y_{d}-y_{o}\right)\right|}
\end{aligned}
$$

where $K_{n}$ is weight ascribed to the importance of a given response by the user, $y_{d}$ is the target response, and $y_{o}$ is response resulting from current set of input parameters. After reproduction, crossover is performed by interchanging the genetic material of two parents. Finally mutation is implemented by randomly changing a fixed number of bits every generation based on specified mutation probability.

\subsection{Neuro-Genetic Synthesis}

The neuro-genetic synthesis methodology has two stages. In the first stage, a neural network model is developed from the measured data. The next stage involves coupling the neural network model with genetic search for subsequent design synthesis and optimization. The neural network does not represent any $\mathrm{RF} /$ microwave component well until it is trained with experimental data. In stage 1, the range of layout parameters is determined within which the desired electrical response of the filter exists. After the range of parameters is determined, experimental design is used to generate the necessary training data using minimum number of experimental trials. The data obtained is used to develop neural network models that relate the layout parameters and electrical response of the filter. The model derived can be used to perform sensitivity analysis or obtain response surfaces. A flow chart of stage 1 of neuro-genetic design is shown in Figure 2. 


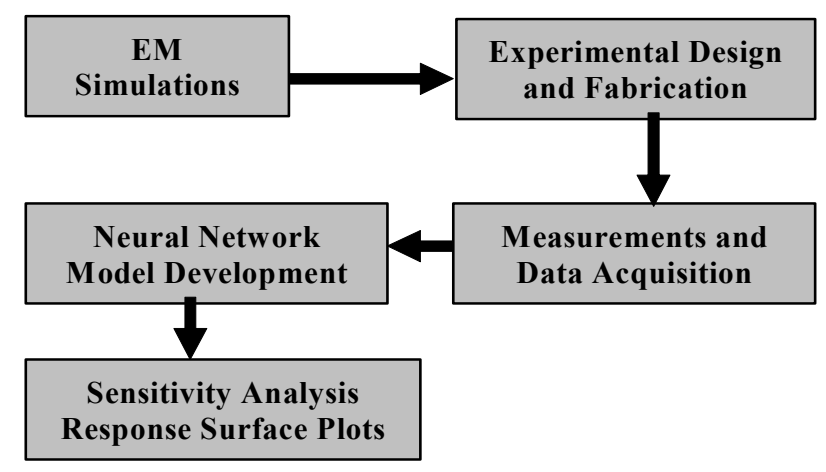

Figure 2. Stage 1: Neural network modeling and analysis.

The second stage is the synthesis stage, where the desired electrical characteristics are provided to a genetic optimizer. The optimizer computes the response of this population using the neural network model and selects the best (i.e., most fit) samples and performs genetic manipulation to obtain results from the best samples. The process continues until the remaining samples produce the set of layout parameters that give (or are closest to) the desired electrical characteristics. The proposed method can assign priority to preferred performance characteristics (through the $K_{n}$ 's in (5)). During filter design, various tradeoffs between electrical design parameters (like bandwidth and insertion loss) can be accounted for using priority assignment during genetic optimization. Due to the flexibility of the genetic approach, the design procedure can be customized to obtain the best available design considering manufacturing and other physical limitations. The flowchart for stage 2 is shown in Figure 3.

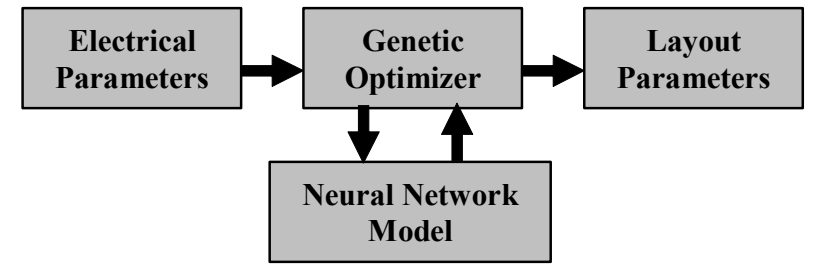

Figure 3. Stage 2: Neuro-genetic filter synthesis.

\section{Neuro-Genetic Design of mm-Wave Front Ends}

The mm-wave front ends selected for neuro-genetic design were low pass and band pass filters. The proposed method was used to design mm-wave low pass filters with cutoff frequencies of 40 and $60 \mathrm{GHz}$ as well as a multilayer LTCC band pass filter at $40 \mathrm{GHz}$.

\subsection{Experimental Design and Modeling: Low Pass Filter}

A prototype low pass filter was designed by combining in cascade the constant- $k, m$-derived sharp cutoff, and $m$-derived matching sections [8]. The filter was designed to obtain a cutoff frequency in the range of $35-70 \mathrm{GHz}$ and an input impedance of $50 \Omega$. The filter was realized using the microstrip line configuration. The layout schematic and parameter ranges are shown in Figure 4 and Table 1, respectively. Latin hypercube sampling was used for experimental design because of its ability to capture non linearity in the design space [9]. The filter was fabricated using a 12-metal-layer LTCC process. A microphotograph of the fabricated LTCC filter samples is shown in Figure 5.

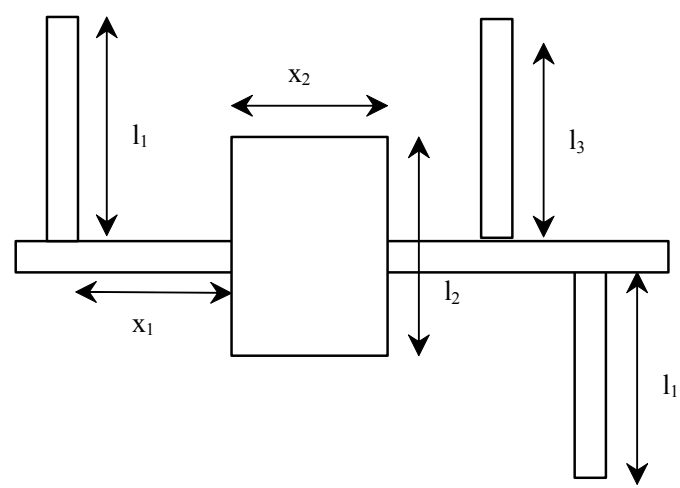

Figure 4. Layout schematic of mm-wave low pass filter.

Table 1: Range of Layout Parameters for Low Pass Filter

\begin{tabular}{|c|c|c|}
\hline $\begin{array}{r}\text { Layout } \\
\text { Parameter }\end{array}$ & $\begin{array}{c}\text { Low } \\
(\mu \mathrm{m})\end{array}$ & $\begin{array}{c}\text { High } \\
(\mu \mathrm{m})\end{array}$ \\
\hline $1_{1}$ & 350 & 550 \\
\hline $1_{2}$ & 400 & 600 \\
\hline $1_{3}$ & 450 & 800 \\
\hline $\mathrm{x}_{1}$ & 300 & 500 \\
\hline $\mathrm{x}_{2}$ & 200 & 400 \\
\hline
\end{tabular}

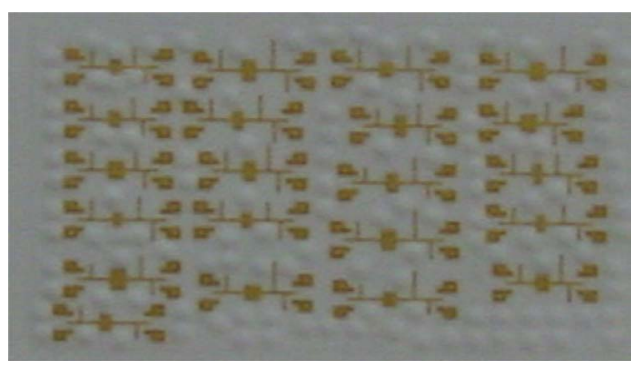
filters.

Figure 5. Fabricated samples of mm-wave low pass

Two-port electrical measurements were performed on the fabricated samples using SOLT calibration and a network analyzer. The measured values of insertion loss for the low pass filters are shown in Figure 6. The measured data was used to obtain neural network models for the cutoff frequency, attenuation, and frequency at the first pole. The learning rate used was 0.001 . Neural network modeling results are shown in Table 2. The derived model exhibited a prediction error less than $5 \%$ for the cutoff frequency and frequency at the first attenuation pole. This accuracy is good, considering the error involved in measurement and parameter extraction at 40-70 GHz. The prediction error for attenuation was large, and this was likely due to errors involved in measurement of rejection losses of order of $-50 \mathrm{~dB}$ at high frequency and calibration errors. 


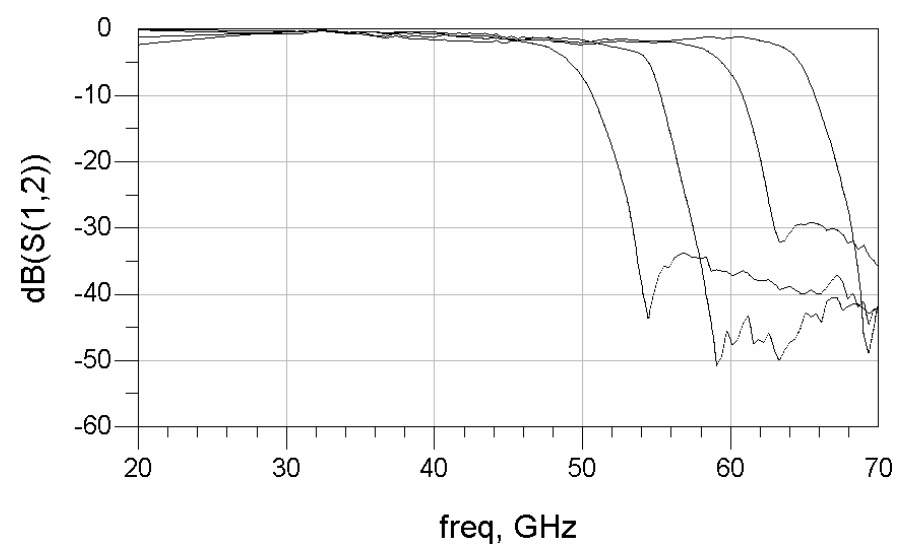

Figure 6. Measured insertion loss of low pass filter samples.

Table 2: Neural Network Modeling of Low Pass Filter

\begin{tabular}{|c|c|c|c|}
\hline Filter Parameter & \multirow{2}{*}{$\begin{array}{c}\text { NN } \\
\text { Structure }\end{array}$} & \multicolumn{2}{|c|}{ Prediction Error } \\
\cline { 3 - 4 } & RMSE & \%RMSE \\
\hline $\begin{array}{c}\text { Cutoff } \\
\text { Frequency }\end{array}$ & $5-7-1$ & 2.7 & 3.9 \\
\hline $\begin{array}{c}\text { Frequency @ } \\
\text { attenuation pole }\end{array}$ & $5-7-1$ & 7.9 & 4.8 \\
\hline $\begin{array}{c}\text { Rejection @ } \\
\text { attenuation pole }\end{array}$ & $5-7-1$ & 25.6 & $21 \%$ \\
\hline
\end{tabular}

\subsection{Experimental Design and Modeling: Bandpass Filter}

One single mode slotted patch filter with a transverse cut on each side was designed and embedded in an LTCC process for $38-40 \mathrm{GHz}$ applications (such as remote sensing and secure communications). The top view of the patch filter designed for a $6.5 \%$ bandwidth, $39 \mathrm{GHz}$ center frequency, and $<3 \mathrm{~dB}$ insertion loss, is shown in Figure 7 . This structure was developed from the basic half-wavelength square patch at $39 \mathrm{GHz}$ by adding a transverse cut $\left(\mathrm{L}_{\mathrm{cl}} \times \mathrm{L}_{\mathrm{cw}}\right)$ on each side. Transverse cuts contribute to significant miniaturization (about 38\%) due to the additional inductance effect. The desired coupling coefficients are obtained by placing the feed lines and the resonator into different vertical metal layers. The layout parameter ranges are shown in Table 3.

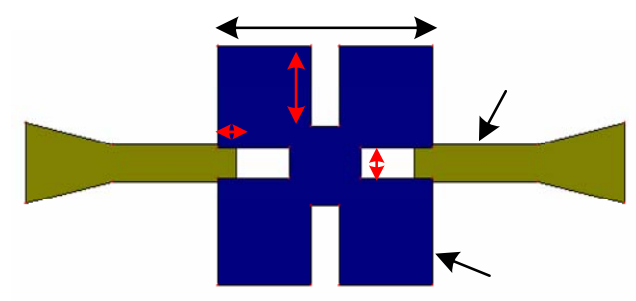

Figure 7. Layout schematic mm-wave bandpass filter.

Table 3: Range of Layout Parameters for Bandpass Filter

\begin{tabular}{|c|c|c|}
\hline $\begin{array}{c}\text { Layout } \\
\text { Parameter }\end{array}$ & $\begin{array}{c}\text { Low } \\
(\mu \mathrm{m})\end{array}$ & $\begin{array}{c}\text { High } \\
(\mu \mathrm{m})\end{array}$ \\
\hline Length $(\mathrm{L})$ & 930 & 1100 \\
\hline $\mathrm{L}_{\mathrm{c} 1}$ & 300 & 400 \\
\hline $\mathrm{L}_{\mathrm{cw}}$ & 90 & 180 \\
\hline $\mathrm{L}_{\text {over }}$ & 50 & 100 \\
\hline
\end{tabular}

The fabricated samples (Figure 8) were measured using the Agilent 8510C Network Analyzer, Cascade Microtech probe station with $250 \mu \mathrm{m}$ pitch air coplanar probes and a standard SOLT calibration method. The experimental samples had center frequencies between $35-42 \mathrm{GHz}$ and $3 \mathrm{~dB}$ bandwidths of 1-4 GHz. The minimum pass band insertion loss was 1-2.5 dB. Sample measured results are shown in Figure 9. The measured data was used to obtain a neural network model (Table 4) for the center frequency, bandwidth, and minimum pass band insertion loss.

Figure 8. Top view of a fabricated mm-wave bandpass filter with an embedded resonator (not visible) and CPW ports on top.

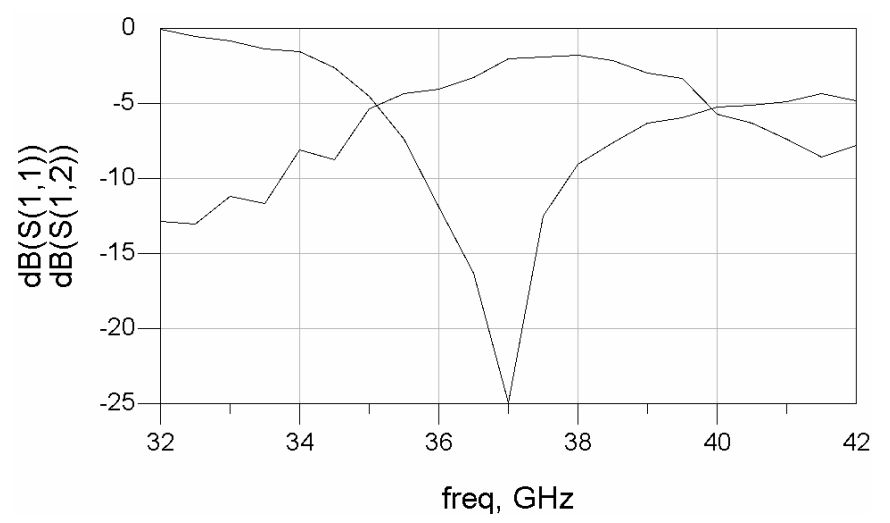

Figure 9. Measured return and insertion loss of bandpass filter.

Table 4: Neural Network Modeling of Bandpass Filter

\begin{tabular}{|c|r|c|c|}
\hline \multirow{2}{*}{$\begin{array}{c}\text { Bandpass Filter } \\
\text { Parameter }\end{array}$} & \multirow{2}{*}{ NN } & \multicolumn{2}{|c|}{ Prediction Error } \\
\cline { 3 - 4 } & Structure & RMSE & \% RMSE \\
\hline Center Frequency & $4-5-1$ & 0.56 & 1.7 \\
\hline Bandwidth & $4-4-1$ & 1.4 & 4.1 \\
\hline Min. Insertion Loss & $4-5-1$ & 2.6 & 10.3 \\
\hline
\end{tabular}

\section{3 mm-Wave Filter Synthesis Results}

The neural network models were used to design LTCC mm-wave low pass and band pass filters using the neurogenetic approach. The genetic algorithm parameters chosen for filter synthesis are shown in Table 4. These parameters were chosen such that the algorithm converged to the desired optimal point with few iterations. Filter synthesis results are shown in Table 6. For the low pass filter, the measured results obtained from the synthesized filter were close to the targeted value for the design at 40 and $60 \mathrm{GHz}$. The rejection at the attenuation pole was not used for synthesis, as it had a very large modeling error. With a small number of experimental runs (16), a low pass filter was synthesized with precise cutoff frequency in the range of $35-65 \mathrm{GHz}$.

However, for the band pass filter, the measured values of the synthesized filters were not as close to the targeted values as expected. This may indicate that the filter layout range should be increased. Furthermore, this was a complicated 
design with single patch, and the filters did not exhibit very good operational characteristics initially. Therefore, the neuro-genetic approach was limited in its ability to synthesize a filter with a narrow bandwidth of $1.2 \mathrm{GHz}$ and insertion loss of $2 \mathrm{~dB}$ with a single patch. A possible method to overcome this limitation would be to widen the layout range and add more patches so that a larger design space could be explored.

Table 5: Genetic Algorithm Parameters

\begin{tabular}{|c|c|}
\hline Genetic Algorithm Parameters & Value \\
\hline Crossover Probability & 0.65 \\
\hline Mutation Probability & 0.01 \\
\hline Population Size & 100 \\
\hline Chromosome Length & 100 \\
\hline
\end{tabular}

Table 5: Mm-Wave Filter Synthesis Results

\begin{tabular}{|c|c|c|c|c|c|c|}
\hline \multicolumn{7}{|c|}{ Mm-wave low pass filter synthesis } \\
\hline & & \multicolumn{2}{|c|}{$\begin{array}{c}\text { Cutoff } \\
\text { Freq. GHz) }\end{array}$} & \multicolumn{2}{|c|}{$\begin{array}{c}\text { Freq. @ atten. } \\
\text { pole }(\mathrm{GHz})\end{array}$} & $\mathrm{l}_{1}, \mathrm{l}_{2}, \mathrm{l}_{3}, \mathrm{x}_{1}, \mathrm{x}_{2}$ \\
\hline & Weight & \multicolumn{2}{|l|}{100} & \multicolumn{2}{|c|}{100} & \\
\hline & Target & \multicolumn{2}{|l|}{40} & \multicolumn{2}{|c|}{45} & \\
\hline 1 & NN-GA & \multicolumn{2}{|c|}{39.70} & \multicolumn{2}{|c|}{47.5} & $\begin{array}{c}417,760,600 \\
375,380\end{array}$ \\
\hline & Target & \multicolumn{2}{|l|}{60} & \multicolumn{2}{|c|}{65} & \\
\hline 2 & NN-GA & \multicolumn{2}{|l|}{59} & \multicolumn{2}{|c|}{66} & $\begin{array}{c}395,500,505, \\
300,220\end{array}$ \\
\hline \multicolumn{7}{|c|}{ Mm-wave bandpass filter synthesis } \\
\hline & & $\begin{array}{l}\text { Center } \\
\text { Freq. } \\
(\mathrm{GHz})\end{array}$ & \multicolumn{2}{|c|}{$\begin{array}{c}\text { 3-dB } \\
\text { Bandwidth } \\
(\mathrm{GHz})\end{array}$} & $\begin{array}{l}\text { Inser. } \\
\text { Loss } \\
(\mathrm{dB})\end{array}$ & $\begin{array}{l}\text { Length, Lc1, } \\
\text { Lcw, Lover }\end{array}$ \\
\hline & Weight & 100 & & 10 & 10 & \\
\hline \multirow[b]{2}{*}{1} & Target & 40 & & 1.2 & 2 & \\
\hline & NN-GA & 41.2 & & 3.2 & 2.88 & $\begin{array}{c}935,315 \\
160,150\end{array}$ \\
\hline
\end{tabular}

\section{Conclusions}

A novel neuro-genetic methodology has been used to synthesize LTCC mm-wave filters. This is the first reported method where measured results were used for synthesis. This method results in more realistic and precise design. The devices obtained from the layout parameters predicted by this method performed within $5 \%$ of the desired targets. This method implements priority to account for design tradeoffs. The method also possesses the potential to be used for designing other passive circuits like baluns, couplers, and antennas, as well as active circuits.

\section{Acknowledgments}

The authors wish to thank the Georgia Electronic Design Center and the National Science Foundation (Grant No. EEC9402723) for support of this research.

\section{References}

1. Gupta, K. C., "Emerging trends in millimeter-wave CAD," IEEE Trans. Microwave Theory \& Tech., vol. 46, (1998) pp. 747-755.

2. Budimir, D., Goussetis, G., "Design of asymmetrical RF and microwave bandpass filters by computer optimization," IEEE Trans. Microwave Theory and Tech., vol. 51, (2003) pp. 1174-1178.

3. Chakravarty, S., and Mittra, R., "Design of microwave filters using a binary coded genetic algorithms," Proc Antennas and Propagation Society International Symposium, July 2000, vol. 1, pp. $144-147$.

4. Pratap, R. J., Sarkar, S., Pinel, S., Laskar, J., and May, G.S., "Modeling and optimization of multilayer RF passives using coupled neural networks and genetic algorithms," 2004 IEEE MTT-S International Microwave Symposium Digest, ,vol.3, (2004), pp. 1557 - 1560.

5. Tong, C., and Sriram, D. Artificial Intelligence in Engineering Design, Academic Press (New York: 1992).

6. Lipmann, R., "An introduction to computing with Neural Nets," IEEE ASSP, Apr. 1987.

7. Goldberg, D., Genetic Algorithms in Search, Optimization \& Machine Learning, Addison Wesley (Mass:1989).

8. Pozar, D. M., Microwave Engineering, Wiley \& Sons, (Amherst, MA:1998).

9. Loh, W. L., "On Latin Hypercube Sampling," The Annals of Statistics vol. 24, (1996) pp.2058-2080. 